\title{
Alterações Musculares e Esqueléticas Cervicais em Mulheres Disfônicas
}

\section{Muscular and Skeletal Changes in Cervical Dysphonic in Women}

\author{
Laiza Carine Maia Menoncin*, Ari Leon Jurkiewicz**, Kelly Cristina A. Silvério ***, \\ Paulo Monteiro Camargo ****, Nathália Martii Monti Wolff*****.
}

\footnotetext{
* Mestre em Distúrbios da Comunicação da Universidade Tuiuti do Paraná. Fisioterapeuta.

** Doutor pela UNIFESP. Docente do Programa de Mestrado em Distúrbios da Comunicação da Universidade Tuiuti do Paraná.

**** Doutora pela Unicamp. Docente do Programa de Mestrado e Doutorado em Distúrbios da Comunicação da Universidade Tuiuti do Paraná.

***** Doutor em Clínica - Cirúrgica pela UFPR. Chefe do Departamento de Laringologia do Hospital Angelina Caron.

****** Médica. Residente Otorrinolaringologia.
}

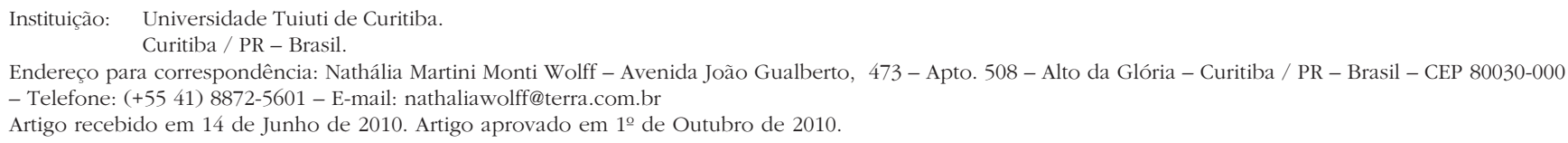

\section{RESUMO}

Introdução:

Objetivo:

Método:

Resultados:

Conclusão:

Palavras-chave:

As alterações vocais e cervicais estão associadas à presença de tensão e de retração muscular cervical. Esses distúrbios comprometem o trato vocal e a região musculoesquelética cervical e, desta forma, podem provocar encurtamentos musculares, dor e fadiga na região cervical e cintura escapular. Avaliar e identificar as alterações cervicais em mulheres com distúrbios vocais, bem como algias cervicais comparando-as a mulheres sem alterações vocais independente de queixas cervicais. Este estudo prospectivo estudou 32 indivíduos no grupo disfônico e 18 indivíduos no grupo controle, idade entre 25 e 55 anos, sexo feminino. Os indivíduos foram submetidos às avaliações, otorrinolaringológica, ortopédica, registro de voz e fisioterápica. Ao Rx da região cervical maior número de pacientes do grupo controle tinham este normal, entretanto, no que se refere à diminuição dos espaços interdiscais os pacientes disfônicos prevaleceram. Além disso, na avaliação postural, a hipercifose da $1^{\underline{a}}$ vértebra torácica ocorreu em 77,0\% do grupo não disfônico ( $\mathrm{p}=0,0091)$, enquanto a rotação cervical esteve presente em 83\% do controle ( $\mathrm{p}=0,0051$ ). Foram identificadas importantes alterações cervicais em ambos os grupos estudados, mas não se pode inferir que as alterações estejam relacionadas diretamente com a disfonia.

\section{SUMMARY} distúrbios da voz, cervicalgia, postura.

Introduction:

Objective:

Method:

The vocal and neck are associated with the presence of tension and cervical muscle contraction. These disorders compromise the vocal tract and musculoskeletal cervical region and, thus, can cause muscle shortening, pain and fatigue in the neck and shoulder girdle.

To evaluate and identify cervical abnormalities in women with vocal disorders, and neck pains comparing them to women without vocal complaints independent of the neck.

This prospective study of 32 subjects studied in the dysphonic group and 18 subjects in the control group, aged between 25 and 55 year old female. The subjects underwent assessments, ENT, orthopedic, physical therapy and voice recording.

Results: At Rx cervical region more patients in the control group had this normal, however, with regard to the reduction of spaces interdiscal dysphonic patients prevailed. Furthermore, postural assessment, the kyphosis of the 1 st thoracic vertebra occurred in $77.0 \%$ of non-dysphonic group $(p=0.0091$ ), while cervical rotation was present in $83 \%$ of control $(p=0.0051)$.

Conclusion: Significant cervical abnormalities in both groups, but cannot be inferred that the changes are directly related to dysphonia.

Keywords: voice disorders, neck pain, posture. 


\section{INTRODUÇÃO}

Há vários anos, estudos científicos vêm demonstrando a teoria da participação da musculatura extrínseca da laringe na função de fonação. Entretanto, alguns estudos colocam em dúvida a participação da musculatura externa da laringe na produção da voz. Paralelamente, diversos trabalhos clínicos evidenciam que as disfonias, em especial as hipercinéticas ou hiperfuncionais, podem estar relacionadas a desequilíbrios da musculatura cervical. Em geral, esse padrão hiperfuncional persiste em situações fonatórias distintas e pode favorecer o aparecimento de lesões orgânicas. Ainda, as alterações orgânicas podem induzir um ajuste funcional inadequado causando disfonia.

Alguns autores consideram que a síndrome da tensão musculoesquelética, também conhecida como disfonia por tensão muscular, trata-se de uma série extensa de alterações vocais não específicas, sendo uma disfonia de difícil definição. Identificam-se duas categorias causais da síndrome da tensão musculoesquelética: a primária, com ausência de alterações estruturais da laringe; e a secundária, com a presença de reações teciduais. À laringoscopia a síndrome da tensão musculoesquelética primária mostra normalidade estrutural e mobilidade das pregas vocais. Já na síndrome da tensão musculoesquelética secundária há presença de lesões laríngeas como nódulos, pólipos e edemas. Desta forma, são observados sinais como: desvios na postura da cabeça e pescoço, hiperextensão da cabeça, pescoço sulcado, enorme tensão na inspiração e insuficiente abertura de boca à emissão. Portanto, há aspectos clínicos relacionados aos quadros de disfonia como as alterações posturais, musculoesqueléticas e a relação do aspecto psicoemocional com a voz.

Desta forma, esta pesquisa visa investigar a relação da disfonia funcional ou orgânica em mulheres com alterações musculares e esqueléticas cervicais.

\section{MÉTOdO}

Participaram deste estudo transversal 50 indivíduos, na faixa etária de 25 a 55 anos, sendo 32 (grupo experimental) com queixas de voz e algia cervical e 18 (grupo controle) sem queixas de voz e independente de algia cervical.

Os critérios de inclusão foram: sexo feminino, presença de queixa de alteração vocal e algia cervical. Os critérios de exclusão foram: doenças cardiovasculares, auto-imunes, traqueobroncopulmonares e diabetes, o uso de antiinflamatórios não hormonais e hormonais, analgésico, colutórios ou outra medicação, bem como condutas terapêuticas que interferissem nas queixas vocais ou cervicais.

Todos os indivíduos assinaram um Termo de Consentimento Livre e Esclarecido (00114/2007), onde obtiveram informações sobre os objetivos, procedimentos que foram efetuados e esclarecimentos sobre as garantias do estudo.

Os indivíduos deste estudo foram selecionados por meio de um questionário previamente elaborado que continha questões objetivas e subjetivas, com respostas únicas, múltiplas e descritivas. Este foi distribuído em escolas públicas e privadas, da Educação Infantil ao Ensino Fundamental, e para pacientes selecionadas por médico ortopedista ou otorrinolaringologista de consultórios privados. O questionário abordou questões sobre a identificação, a atividade profissional, a presença ou não de queixa vocal, as sensações laríngeas, a queixa de dor no pescoço e nos ombros, bem como associação de dor com disfonia. Inicialmente, foram recolhidos 80 questionários que permitiram a seleção de 50 indivíduos, sendo 32 (grupo experimental) com queixas de voz e algia cervical e 18 (grupo controle).

Todos os indivíduos passaram por avaliação otorrinolaringológica (ORL), ortopédica, fisioterápica e registro vocal. A avaliação ORL consistiu de realização de videolaringoestroboscopia realizada por um especialista para elucidação diagnóstica. A avaliação ortopédica foi realizada por especialista, que, embasado nas queixas de algia cervical aplicou testes de mobilidade cervical nos movimentos de flexão, extensão, rotação lateral direita e esquerda, inclinação lateral de forma ativa, testes específicos de tração, compressão, manobra de Valsalva e encurtamentos musculares, bem como a palpação em partes moles. A coluna cervical foi investigada através de raios X simples, nas incidências de perfil, anteroposterior e pósteroanterior. A avaliação fisioterápica constou de exame de inspeção postural, em que o indivíduo posicionou-se diante de um quadro postural para a visibilização dos perfis anterior, lateral e posterior. Na sequência eram palpados os músculos trapézio (fibras superiores), esternocleidomastóideos, escalenos (fibras anterior, média e posterior), linha nucal inferior bilateral e a "base da nuca", com o intuito de identificar "pontos-gatilho" e presença de dor. Em seguida, foram realizados testes de encurtamentos para os músculos trapézio (fibras superiores), esternocleidomastóideos e eretores da coluna vertebral. O registro de voz propiciou a avaliação perceptivo-auditiva para classificação dos indivíduos em grupo disfônico e não disfônico. Para registro das vozes utilizou-se um microfone ultimate headset unidirecional condenser profissional, estéreo, da marca Plantronics áudio $90^{\circledR}$; acoplado a um computador notebook Toshiba modelo A135-S4737; e do software Multi Speech - MDVP da Kay Elemetrics ${ }^{\circledR}$, pertencentes ao 
Tabela I. Sensações laríngeas relatadas pelos indivíduos dos grupos disfônico e não disfônico.

\begin{tabular}{|c|c|c|c|}
\hline $\begin{array}{l}\text { Sensações } \\
\text { Laríngeas }\end{array}$ & $\begin{array}{c}\text { Grupo } \\
\text { Disfônico } \\
(\mathrm{N}=32)\end{array}$ & $\begin{array}{c}\text { Grupo Não } \\
\text { Disfônico } \\
(\mathrm{N}=\mid 8)\end{array}$ & $\mathrm{p}$ \\
\hline Irritação & $19(59,0 \%)$ & $2(11,0 \%)$ & $* 0,00 \mid 8$ \\
\hline Secura & $18(56,0 \%)$ & - & $* 0,0002$ \\
\hline Pigarro & II (34,0\%) & | (5,5\%) & $* 0,0275$ \\
\hline Prurido & II (34,0\%) & I (5,5\%) & $* 0,0275$ \\
\hline Dornagarganta & $8(25,0 \%)$ & - & $* 0,0250$ \\
\hline Ausência & $5(15,0 \%)$ & $16(88,0 \%)$ & $* 0,0000$ \\
\hline
\end{tabular}

Legenda: $\mathrm{N}=$ número de indivíduos. * Há diferença estatística significativa pelo Teste de Diferença de Proporções.

laboratório de voz da Universidade Tuiuti do Paraná. O indivíduo foi orientado a falar espontaneamente por 30 segundos, respondendo a pergunta: "O que você acha da sua voz" respirando naturalmente e no seu ritmo de fala, com pitch e loudness habitual. Os indivíduos repetiram esta emissão por três vezes, com intervalo de 30 segundos entre as gravações. A análise perceptivo-auditiva foi realizada por três fonoaudiólogas, em duplo cego, por consenso, com amostra da fala espontânea e consistiu da classificação do tipo de voz, baseada na escala GRBAS - proposta por Hirano (1981).

Os resultados, isolados e associados, foram analisados pelo Teste de Diferença de Proporções, Teste de Quiquadrado, Teste de Fischer e Teste de Mann-Whitney, considerando-se o nível de significância de 0,05\%.

\section{RESULTADOS}

Os resultados relativos às sensações laríngeas, investigadas através de um questionário, demonstraram que, nos pacientes com disfonia, $59 \%$ apresentaram irritação, $56 \%$ secura, 34\% de pigarro e prurido, dor de garganta em $25 \%$ e $15 \%$ não relatavam queixas (Tabela 1) com uma diferença estatisticamente significativa em comparação com o grupo não disfônico $(\mathrm{p}<0,005)$.

Em relação à avaliação da dor no pescoço e ombros os resultados estão na Tabela 2. Em adição, a Tabela 3 mostra a relação entre os indivíduos avaliados e o diagnóstico clínico laríngeo.

A avaliação do raio X de coluna cervical demonstrou diferença estatística na comparação entre os grupos no que se refere à diminuição de espaços interdiscais, alterações mais frequentes nos indivíduos disfônicos e, inversamente, raio-x cervical normal, sendo mais prevalente no grupo controle (Tabela 4).
Tabela 2. Distribuição de indivíduos dos grupos disfônico e não disfônico de acordo com a queixa de dor no pescoço e ombros e associação com queixas relacionadas à laringe.

\begin{tabular}{lccc}
\hline Dor & $\begin{array}{c}\text { Grupo } \\
\text { Disfônico } \\
(\mathrm{N}=32)\end{array}$ & $\begin{array}{c}\text { Grupo Não } \\
\text { Disfônico } \\
(\mathrm{N}=\mid 8)\end{array}$ & $p$ \\
\hline $\begin{array}{l}\text { Dor no pescoço } \\
\text { ou ombros } \\
\begin{array}{l}\text { Dor no pescoço } \\
\text { ou ombros e }\end{array}\end{array}$ & $29(90,0 \%)$ & $17(94,0 \%)$ & 0,6297 \\
$\begin{array}{l}\text { rouquidão } \\
\text { Sem dor }\end{array}$ & $6(18,0 \%)$ & - & 0,0616 \\
\hline
\end{tabular}

Legenda: $\mathrm{N}=$ número de indivíduos

Tabela 3. Distribuição de indivíduos dos grupos disfônico e não disfônico quanto ao diagnóstico clínico laríngeo.

\begin{tabular}{|c|c|c|c|}
\hline $\begin{array}{l}\text { Diagnóstico } \\
\text { Laríngeo }\end{array}$ & $\begin{array}{c}\text { Grupo } \\
\text { Disfônico } \\
(\mathrm{N}=32)\end{array}$ & $\begin{array}{c}\text { Grupo Não } \\
\text { Disfônico } \\
(\mathrm{N}=18)\end{array}$ & $\mathrm{p}$ \\
\hline \multicolumn{4}{|l|}{ Fendatriangular } \\
\hline posterior & $7(21,0 \%)$ & $7(38,0 \%)$ & 0,0709 \\
\hline \multicolumn{4}{|l|}{ Fendatriangular } \\
\hline médio-posterior & $7(21,0 \%)$ & $7(38,0 \%)$ & 0,0709 \\
\hline $\begin{array}{l}\text { Fenda ampulhetae } \\
\text { nódulo }\end{array}$ & $3(9,0 \%)$ & | (5,5\%) & 0,6586 \\
\hline $\begin{array}{l}\text { Cisto + fenda em } \\
\text { ampulheta + nódulo }\end{array}$ & $3(9,0 \%)$ & - & 0,1961 \\
\hline $\begin{array}{l}\text { Fendatriangular } \\
\text { médio-posteriore } \\
\text { nódulo }\end{array}$ & $2(6,0 \%)$ & - & 0,2945 \\
\hline Paquidermia + cisto & $2(6,0 \%)$ & - & 0,2945 \\
\hline Vasculodisgenesia & | (3,0\%) & - & 0,4617 \\
\hline Sulco & | (3,0\%) & - & 0,4617 \\
\hline Pólipo & | (3,0\%) & - & 0,4617 \\
\hline Laringitesupra-glótica & I (3,0\%) & - & 0,4617 \\
\hline $\begin{array}{l}\text { Paquidermia + } \\
\text { Fendatriangular } \\
\text { posterior }\end{array}$ & | (3,0\%) & I (5,5\%) & 0,6091 \\
\hline $\begin{array}{l}\text { Paquidermia + } \\
\text { rimatriangular } \\
\text { médio-posterior }\end{array}$ & | (3,0\%) & - & 0,4617 \\
\hline Paquidermia & | (3,0\%) & - & 0,4617 \\
\hline Rimafusiforme & | (3,0\%) & - & 0,4617 \\
\hline Normal & - & $2(11 \%)$ & 0,0615 \\
\hline
\end{tabular}

Legenda: $\mathrm{N}=$ número de indivíduos. 
Tabela 4. Distribuição de indivíduos dos grupos disfônico e não disfônico quanto ao diagnóstico radiológico simples da coluna cervical.

\begin{tabular}{lccc}
\hline $\begin{array}{l}\text { Diagnóstico } \\
\text { Vertebral }\end{array}$ & $\begin{array}{c}\text { Grupo } \\
\text { Disfônico } \\
(\mathrm{N}=32)\end{array}$ & $\begin{array}{c}\text { Grupo Não } \\
\text { Disfônico } \\
(\mathrm{N}=\mid 8)\end{array}$ & $\mathrm{P}$ \\
\hline
\end{tabular}

Diminuição de

espaços interdiscais

$14(43,0 \%)$

| (5,5\%)

$* 0,0075$

Osteófitos

$4(12,0 \%)$

$2(11,0 \%)$

0,9162

Osteófitos e

diminuição de

espaçosinterdiscais I (3,0\%) $\quad-\quad 0,4617$

Examenormal $\quad 13(40,0 \%) \quad \mid 5(83,0 \%) \quad * 0,005$ |

Legenda: $\mathrm{N}$ = número de indivíduos. *Há diferença estatística significante pelo Teste de Diferença de Proporções.

Tabela 5. Distribuição de indivíduos dos grupos disfônico e não disfônico quanto à avaliação postural Vista anterior de coluna cervical.

\begin{tabular}{lccc}
\hline $\begin{array}{l}\text { Avaliação Postural } \\
\text { Anterior }\end{array}$ & $\begin{array}{c}\text { Grupo } \\
\text { Disfônico } \\
(\mathrm{N}=32)\end{array}$ & $\begin{array}{c}\text { Grupo Não } \\
\text { Disfônico } \\
(\mathrm{N}=\mid 8)\end{array}$ & $P$ \\
\hline $\begin{array}{l}\text { Rotação cervical } \\
\text { Desnivelamento }\end{array}$ & $20(62,0 \%)$ & $15(83,0 \%)$ & $* 0,005$ | \\
de ombros & $17(53,0 \%)$ & $15(83,0 \%)$ & 0,3930 \\
Inclinaçãolateral & $12(37,0 \%)$ & $4(22,0 \%)$ & 0,2789 \\
Semalterações & $\mid(3,0 \%)$ & $\mid(5,5 \%)$ & $0,609 \mid$ \\
\hline
\end{tabular}

Legenda: $\mathrm{N}=$ número de indivíduos. * Há diferença estatística significativa pelo Teste de Diferença de Proporções.

Analisando-se os dados posturais, referentes à face anterior da coluna cervical nos pacientes disfônicos, $62 \%$ apresentaram rotação cervical, sendo que no grupo de pacientes não disfônicos este distúrbio foi observado em 83\%. (Tabela 5). Ainda, a apreciação da coluna cervical lateral no grupo dos disfônicos, hipercifose de T1 em 37\%, enquanto no grupo de pacientes não disfônicos encontrouse $77 \%$, com resultados estatisticamente significativos (Tabela 6).

Os resultados quanto à presença de dor à palpação do pescoço e músculo trapézio (fibras superiores) está demonstrado na Tabela 7, porém os resultados não são estatisticamente significativos.

Finalizando, quanto à identificação de pontos de gatilho em região de coluna cervical (Tabela 8) e ainda, na
Tabela 6. Distribuição de indivíduos dos grupos disfônico e não disfônico quanto à avaliação postural Vista lateral de coluna cervical.

\begin{tabular}{lccc}
\hline $\begin{array}{l}\text { Avaliação Postural } \\
\text { Lateral }\end{array}$ & $\begin{array}{c}\text { Grupo } \\
\text { Disfônico } \\
(\mathrm{N}=32)\end{array}$ & $\begin{array}{c}\text { Grupo Não } \\
\text { Disfônico } \\
(\mathrm{N}=18)\end{array}$ & $\mathrm{P}$ \\
\hline
\end{tabular}

Anteroversão

cenvical $\quad 23(71,0 \%) \quad 16(88,0 \%) \quad 0,1759$

Hipercifose da

primeiratorácica

\begin{tabular}{lccc}
$(\mathrm{TI})$ & $12(37,0 \%)$ & $14(77,0 \%)$ & $* 0,009 \mid$ \\
$\begin{array}{l}\text { Retificação cervical } \\
\text { Hiperlordose }\end{array}$ & $5(15,0 \%)$ & - & 0,0904 \\
\begin{tabular}{l} 
cervical \\
\hline
\end{tabular} & $4(12,0 \%)$ & $2(11,0 \%)$ & 0,9162 \\
\hline
\end{tabular}

Legenda: $\mathrm{N}$ = número de indivíduos. * Há diferença estatística significativa pelo Teste de Diferença de Proporções.

Tabela 7. Distribuição de indivíduos dos grupos disfônico e não disfônico quanto ao local da presença de dor na palpação do pescoço e músculo trapézio (fibras superiores).

\begin{tabular}{lccc}
\hline $\begin{array}{l}\text { Presença de Dor } \\
\text { àPalpação }\end{array}$ & $\begin{array}{c}\text { Grupo } \\
\text { Disfônico } \\
(\mathrm{N}=32)\end{array}$ & $\begin{array}{c}\text { Grupo Não } \\
\text { Disfônico } \\
(\mathrm{N}=18)\end{array}$ & $\mathrm{P}$ \\
\hline $\begin{array}{l}\text { Músculo trapézio } \\
\text { (fibras superiores) }\end{array}$ & $26(81,0 \%)$ & $14(77,0 \%)$ & 0,7378 \\
$\begin{array}{l}\text { "Plano de nuca" e } \\
\text { linhanucal inferior } \\
\text { bilateral }\end{array}$ & $10(31,0 \%)$ & $8(44,0 \%)$ & 0,3616 \\
$\begin{array}{l}\text { Músculo esterno- } \\
\text { cleidomastoideo }\end{array}$ & $3(9,0 \%)$ & - & $0,196 \mid$ \\
Ausência de dor & $4(12,0 \%)$ & $4(22,0 \%)$ & 0,3543 \\
\hline
\end{tabular}

Legenda: $\mathrm{N}=$ número de indivíduos.

avaliação dos encurtamentos musculares da coluna cervical (Tabela 9) não se mostrou diferenças entres os dois grupos.

\section{DISCUSSÃO}

A mulher, indivíduo eleito nesta pesquisa, apresenta o maior número de queixas de distúrbios vocais, pois falam com maior intensidade e frequência, bem como utilizam a vOZ como recurso profissional. Além disso, apresentam uma anatomia laríngea com dimensão reduzida colaborando, juntamente com a voz mais aguda, para o desenvolvimento de alterações vocais e cervicais.

No presente estudo foi relatado, através de uma entrevista inicial, a presença de sintomas laríngeos em ambos os grupos disfônico e não disfônico. Essas sensações 
Tabela 8. Distribuição de indivíduos dos grupos disfônico e não disfônico quanto à identificação de ponto Gatilho em região de coluna cervical.

\begin{tabular}{|c|c|c|c|}
\hline Ponto Gatilho & $\begin{array}{c}\text { Grupo } \\
\text { Disfônico } \\
(\mathrm{N}=32)\end{array}$ & $\begin{array}{c}\text { Grupo Não } \\
\text { Disfônico } \\
(\mathrm{N}=\mid 8)\end{array}$ & $p$ \\
\hline $\begin{array}{l}\text { Músculotrapézio } \\
\text { (bilateral) }\end{array}$ & $28(87,0 \%)$ & $16(88,0 \%)$ & 0,9191 \\
\hline $\begin{array}{l}\text { Músculotrapézio } \\
\text { (hemidireito) }\end{array}$ & $3(9,0 \%)$ & I (5,5\%) & 0,6586 \\
\hline $\begin{array}{l}\text { Músculotrapézio } \\
\text { (hemiesquerdo) }\end{array}$ & $3(9,0 \%)$ & I (5,5\%) & 0,6586 \\
\hline Músculos escalenos & I (3,0\%) & - & 0,4617 \\
\hline $\begin{array}{l}\text { Ausência de ponto } \\
\text { gatilho }\end{array}$ & I (3,0\%) & - & 0,4617 \\
\hline
\end{tabular}

Legenda: $\mathrm{N}$ = número de indivíduos.

laríngeas geram desconforto no ato de falar e de deglutir, impedindo o indivíduo de ter boa produção vocal e comunicação viável e clara, causando perturbações no trabalho e até mesmo no ambiente social. A associação das sensações laríngeas e queixas vocais provocam um intenso distúrbio vocal o qual se denomina disfonia.

Independente da presença de alterações vocais, as mulheres estão predispostas à presença de dores, desconfortos cervicais e fadiga muscular na região cervical. Entretanto, não há achados na literatura que fazem referência quanto ao sintoma de dor em coluna cervical em indivíduos disfônicos.

Paralelamente, durante a avaliação videolaringoscópica demonstrou-se que o diagnóstico de rima glótica triangular posterior e médio-posterior é bastante frequente e considerado fisiológico quando se trata de rima glótica feminina. No entanto, na avaliação perceptivo-auditiva notou-se a presença de alteração vocal, porém sem lesões de pregas vocais. Esses dados levam a crer nas causas mais comuns de disfonia como o mau uso da voz ou abuso vocal (1).

Ainda, os indivíduos da pesquisa foram submetidos a exame de imagem radiológica simples com o intuito de identificar lesões ósseas e definir os motivos das algias cervicais e dos ombros. É possível que os desequilíbrios musculares e ósseos como o aumento da cifose torácica ocasionados por maus hábitos posturais,bem como a falta de ergonomia, as retrações musculares, os traumas diretos e indiretos, o estresse físico e emocional, os fatores ambientais e até mesmo sedentarismo sejam as causas primordiais dessas algias, sendo pouco prováveis as causas de origem óssea, discordando dos resultados encontrados na literatura, a qual cita a artrose como maior causa das algias cervicais (2).
Tabela 9. Distribuição de indivíduos dos grupos disfônico e não disfônico quanto ao encurtamento muscular da coluna cervical.

\begin{tabular}{lccc}
\hline $\begin{array}{l}\text { Encurtamento } \\
\text { Muscular }\end{array}$ & $\begin{array}{c}\text { Grupo } \\
\text { Disfônico } \\
(\mathrm{N}=32)\end{array}$ & $\begin{array}{c}\text { Grupo Não } \\
\text { Disfônico } \\
(\mathrm{N}=\mid 8)\end{array}$ & $p$ \\
\hline $\begin{array}{l}\text { Músculotrapézio } \\
\text { (fibrassuperiores) }\end{array}$ & $31(96,0 \%)$ & $18(100,0 \%)$ & 0,3943 \\
$\begin{array}{l}\text { Músculo esterno- } \\
\text { cleidomastoideo }\end{array}$ & $25(78,0 \%)$ & $16(88,0 \%)$ & 0,3854 \\
$\begin{array}{l}\text { Músculoseretores } \\
\text { dacoluna }\end{array}$ & $8(25,0 \%)$ & $4(22,0 \%)$ & $0,8 \mid 24$ \\
\hline
\end{tabular}

Legenda: $\mathrm{N}=$ número de indivíduos.

No presente estudo, foram observados e identificados importantes alterações na região cervical tanto em mulheres portadoras de disfonia quanto nas sem alterações vocais. Entretanto, houve um maior número de pacientes com alterações posicionais no grupo não disfônico, não se podendo atribuir, portanto, a disfonia como causa desencadeante de alteração postural. Convém ressaltar que não foram encontradas diferenças quanto ao desnivelamento dos ombros, discordando de um estudo realizado, o qual encontrou um desnivelamento dos ombros nas pacientes disfônicas (3).

Os pontos-gatilho constituem-se em nódulos musculares palpáveis, a maior parte dolorosos, originados após pequenos traumas diretos ou indiretos que produzem lesões nas fáscias musculares. Neste estudo foi detectada a presença considerável de pontos-gatilho, em especial na região de trapézio (fibras superiores), de forma similar nos dois os grupos. Não foram encontradas referências sobre a presença de pontos-gatilho na literatura em mulheres disfônicas.

Paralelamente, identificaram-se importantes retrações musculares em ambos os grupos, entretanto essas alterações independem do uso inadequado da voz. Além disso, este estudo demonstrou que as mulheres do grupo disfônico apresentaram encurtamento no músculo esternocleidomastoideo e no músculo trapézio (fibras superiores), concordando com dados da literatura (4). É importante lembrar que a maioria das pacientes apresentam um quadro de disfonia funcional devido ao abuso vocal ou mau uso da voz, podendo ser proveniente do nível de tensão muscular, bem como de encurtamentos na região cervical e, dessa forma, o exame laringoscópico não evidencia alterações estruturais características (5). O aumento no tônus em toda a musculatura laríngea intrínseca e extrínseca simultaneamente, associadas à presença de 
fenda glótica posterior e laringe elevada definem a disfonia por tensão muscular $(6,7,8,9,10)$. Ainda, estudos relatam que uma fonação constantemente associada a uma postura laríngea inadequada pode levar a mudanças orgânicas como nódulos ou pólipos, particularmente em mulheres, com a presença de fenda glótica posterior, associando-se ao aumento na tensão laríngea e mais diretamente ao desequilîbrio do músculo cricoaritenóideo posterior durante a fonação $(8,11)$. Os resultados do presente estudo concordam com a literatura, já que a maioria das participantes disfônicas apresentaram uma disfonia importante na avaliação auditivo-perceptiva, bem como encurtamentos musculares na região cervical, porém, o diagnóstico pela videolaringoestroboscopia não apresentaram lesão nas pregas vocais (8). O aumento da lordose cervical e/ou lombar, o aumento da cifose torácica e/ou alterações da posição da cabeça podem gerar compensações na região cervical e também na laringe $(12,13)$.

Apesar de muitas evidências, coloca-se em questão a relação da disfonia com a participação da musculatura cervical externa ou paralaríngea (11), já que mulheres não disfônicas apresentam tantas alterações quanto à disfônicas, contribuindo para a teoria que coloca em dúvida a participação da musculatura externa laríngea na produção vocal.

Sobretudo, esta pesquisa mostrou-se valiosa e surpreendente quanto à investigação e detecção de alterações posturais tanto em mulheres com e sem alterações vocais. Ao passo que contribui para o tratamento multidisciplinar (otorrinolaringologia, ortopedia, fonoaudiologia e fisioterapia), bem como a continuidade do mesmo para obter melhores benefícios e informações à sociedade.

\section{CONCLUSÃO}

O presente estudo demonstrou que foram identificadas importantes alterações cervicais em mulheres com ou sem disfonia, não sendo possível determinar uma relação de causalidade entre elas.

\section{ReFERÊNCIA BiBLIOGRÁFICA}

1. Greene MCL. Distúrbios da voz. 4a. ed. São Paulo: Manole; 1989.

2. Knoplich J. Enfermidades da Coluna Vertebral. 2a ed. São Paulo: Panamed; 1986.
3. Nelli EA. Estudo da postura corporal em portadores de disfonia [dissertação]. Bauru (SP): Universidade de São Paulo; 2006.

4. Anelli W, Xavier C. Novo enfoque de atendimento a pacientes disfônicos em instituição: grupos de orientação In: Marchezan IQ, Bolaffi C, Gomes ICD, Zorzi JL. Tópicos em Fonoaudiologia. São Paulo: Lovise; 1995. p. 331-347.

5. Imamura R, Tsuji DH. Disfonia espasmódica de adução, tremor vocal e disfonia de tensão muscular: é possível fazer o diagnóstico diferencial? RevBras Otorrinolaringol. 2006, 72(4):434.

6. Aronson AE, Brown JR, Litin EM, Pearson JS. Spastic dysphonia. II. Comparison with essential (voice) tremor and other neurologic and psychogenic dysphonias. J Speech Hear Disord. 1968, 33(3):219-31.

7. Barkmeier JM, Case JL. Differential diagnosis of adductortype spasmodic dysphonia, vocal tremor, and muscle tension dysphonia. Curr Opin Otolaryngol Head Neck Surg. 2000, 8(3):174-9.

8. Morrison MD, Rammage LA, Belisle GM, Pullan CB, Nichol H. Muscular tension dysphonia. The Journal of Otolaryngology. 1983, 12(5):302-6.

9. Aronson AE. Organic voice disorders: neurologic disease. In: Clinical voice disorders. New York: Thieme Inc.;1985. p. $76-125$.

10. Colton RH, Casper JK. Compreendendo os problemas de voz: uma perspectiva fisiológica ao diagnóstico e ao tratamento. Porto Alegre: Artes Médicas; 1996. p. 3-215.

11. Angsuwarangsee T, Morrison M. Extrinsic laryngeal muscular tension in patients with voice disorders. J Voice. 2002, 16:333-43.

12. Grini MN, Oaknine M, Giovanni A. Contemporary postural and segmental modification of forced voice. Rev. Laryngol Otol Rhinol. 1998, 119(4):253-7.

13. Hsiung MW, Hsiao YC. The Characteristic Features of Muscle Tension Dysphonia before and after Surgery in Benign Lesions of the Vocal Fold. ORL. 2004, 66:246-254. 